\title{
1 A wheat kinase and immune receptor form the host-specificity 2 barrier against the blast fungus
}

3 Sanu Arora ${ }^{1,8}$, Andrew Steed $^{1,8}$, Rachel Goddard ${ }^{1,8}$, Kumar Gaurav $^{1,8}$, Tom O’Hara ${ }^{1,8}$, Adam

4 Schoen $^{3}$, Nidhi Rawat ${ }^{3}$, Ahmed F. Elkot ${ }^{5}$, Catherine Chinoy ${ }^{1}$, Martha H. Nicholson ${ }^{1}$, Soichiro

5 Asuke $^{4}$, Burkhard Steuernagel ${ }^{1}$, Guotai $\mathrm{Yu}^{1}$, Rajani Awal ${ }^{1}$, Macarena Forner-Martínez ${ }^{1}$,

6 Luzie Wingen ${ }^{1}$, Erin Baggs ${ }^{6}$, Jonathan Clarke ${ }^{1}$, Ksenia V. Krasileva ${ }^{6}$, Yukio Tosa ${ }^{4}$, Jonathan

7 D. G. Jones ${ }^{2}$, Vijay K. Tiwari ${ }^{3}$, *Brande B. H. Wulff ${ }^{1,7}$, *Paul Nicholson ${ }^{1}$

$8 \quad{ }^{1}$ John Innes Centre, Norwich Research Park, Norwich, UK.

$9{ }^{2}$ The Sainsbury Laboratory, Norwich Research Park, Norwich, UK.

$10{ }^{3}$ Department of Plant Science and Landscape Architecture, University of Maryland, College

11 Park, MD 20742, United States.

$12{ }^{4}$ Graduate School of Agricultural Science, Kobe University, Kobe 657 $\square$ 8501, Japan.

$13{ }^{5}$ Wheat Research Department, Field Crops Research Institute, Agricultural Research Center, 14 12619, Giza Egypt.

$15{ }^{6}$ Department of Plant and Microbial Biology, University of California, Berkeley, California 1694720, USA.

$17{ }^{7}$ Present address: Center for Desert Agriculture, Biological and Environmental Science and 18 Engineering Division (BESE), King Abdullah University of Science and Technology 19 (KAUST), Thuwal 23955-6900, Saudi Arabia.

$20 \quad{ }^{8}$ These authors contributed equally.

21 *Correspondence should be addressed to PN (paul.nicholson@jic.ac.uk) or BBHW 22 (brande.wulff@kaust.edu.sa)

\section{Abstract}

24 Since emerging in Brazil in 1985, wheat blast has spread throughout South America and recently appeared in Bangladesh and Zambia. We show that two wheat resistance genes, $R w t 3$ and $R w t 4$, acting as host-specificity barriers against non-Triticum blast pathotypes encode a nucleotide-binding leucine-rich repeat immune receptor and a tandem kinase, respectively. Molecular isolation of these genes allowed us to develop assays that will ensure the inclusion of these two genes in the wheat cultivars to forestall the recurrence of blast host 30 jumps. 


\section{Main}

34 The occurrence of pathogen host jumps suggests that seemingly durable non-host resistance 35 can be fragile ${ }^{1}$. This is illustrated by the jump of the blast fungus (Pyricularia oryzae, syn.

36 Magnaporthe oryzae) from ryegrass to wheat in Brazil in $1985^{2}$. The pathogen subsequently 37 spread to cause epidemics in other regions of Brazil and neighbouring countries including,

38 Bolivia and Paraguay ${ }^{3}$. Outbreaks of wheat blast occurred in Bangladesh in 2016 and the 39 disease was reported from Zambia in $2018^{4,5}$. Wheat blast is now considered to pose a threat

40 to global wheat production ${ }^{6}$, and discovery and deployment of resistance genes against this 41 pathogen are critical to mitigate its threat.

42 While Pyricularia oryzae exhibits a high level of host specificity, Triticum pathotypes are 43 closely related to Lolium and Avena pathotypes ${ }^{7}$. Two pathogen genes, PWT3 and PWT4, 44 condition avirulence of Avena pathotypes on wheat (Triticum aestivum) while PWT3 prevents 45 infection of wheat by Lolium pathotypes ${ }^{8,9}$.

46 The resistance genes Rwt3 and Rwt4 in wheat recognise respectively the PWT3 and PWT4 47 avirulence gene products to prevent infection. It has been proposed that the epidemics in 48 Brazil occurred due to the widespread cultivation of varieties lacking $R w t 3$ that are 49 susceptible to Lolium pathotypes ${ }^{7}$. Lolium pathotypes have also been associated with the 50 occurrence of wheat blast in the $\mathrm{USA}^{10,11}$. These reports emphasize the importance of 51 maintaining Rwt3 and Rwt4 in wheat cultivars to prevent future host jumps of the Avena, 52 and/or Lolium pathotypes.

53 To identify candidates for $R w t 3$ and $R w t 4$, we used a Triticeae bait library (Table S1, 54 Additional File F1) to capture and sequence the NLR complements of 320 wheat lines 55 including 300 wheat landraces from the A.E. Watkins collection harbouring the genetic 56 diversity existing prior to intensive breeding (Table S2, Supplementary Fig. 1). We screened 57 seedlings of the panel with Br48, a Triticum pathotype of Pyricularia oryzae, transformed 58 with either PWT3 or $P W T 4^{7}$ (Table S3; Supplementary Fig. 2, 3) and performed $k$-mer based 59 association genetics. This led to an identification of candidate NLR genes for PWT3 and 60 PWT4 recognition (Fig 1a-b, Supplementary Fig. 4, Supplementary Fig. 11) on chromosome $611 \mathrm{D}$ within the mapping intervals of $R w t 3$ and $R w t 4$, respectively ${ }^{9,12}$.

62 Investigating the presence of these candidate genes in the NLR assemblies of Aegilops 63 tauschii $^{13}$, the D-genome progenitor of bread wheat, the Rwt4 candidate was found only in 64 lineage 2 (L2) while the Rwt3 candidate was found only in lineage 1 (L1) (Table S4, 
65 Supplementary Fig. 5). This explains why we could identify only the Rwt4 candidate, and not

66 the Rwt3 candidate, by phenotyping and performing association genetics on an NLR gene

67 enrichment-sequenced Ae. tauschii L2 panel $^{13}$ (Table S5, Supplementary Fig. 6, 7). The L2

68 origin of Rwt4 is consistent with L2 being the major contributor of the wheat D-genome,

69 however, the L1 origin of $R w t 3$ is more remarkable considering that the L1 signature in wheat

70 is mostly concentrated around a $5 \mathrm{Mb}$ region surrounding the Rwt3 candidate ${ }^{14}$

71 (Supplementary Fig. 8). This finding suggests that pathogen pressure could have played a

72 significant role in post-domestication wheat evolution.

73 To functionally validate the $R w t 3$ NLR candidate, we screened a TILLING population of 74 Jagger $^{15}$ and found three lines each carrying a functional mutation in this gene 75 (Supplementary Fig. 9). One line, M217, is homozygous for a mutation causing a premature 76 stop codon whereas another, M698, is homozygous for a mis-sense mutation (G241E) 77 predicted to cause functional aberration in the protein (Fig. 1c, Table S6). In both the leaf and 78 head assays of these mutants using $\mathrm{Br} 48+P W T 3$, a loss of the wildtype resistance was 79 observed (Fig. 1d-e). The third line, M1164, is heterozygous for another deleterious mis80 sense mutation (E492K) (Fig. 1c, Table S6). In both the leaf and head assays of the 81 segregating progeny of M1164 using Br48+PWT3, those homozygous for the mutation were 82 found to be susceptible while the others were resistant (Fig 1d-e, Supplementary Fig. 10).

83 The clear loss of function observed in three independently derived TILLING mutants and the 84 co-segregation of the M1164 mutation with susceptibility shows that the Rwt3 NLR candidate 85 is required for resistance to $P$. oryzae expressing the $P W T 3$ effector.

86 We observed that the identified Rwt4 NLR candidate is adjacent to an allele of a wheat 87 tandem kinase (WTK) previously reported to confer resistance against powdery mildew ${ }^{16}$.

88 The $532 \mathrm{~kb}$ mapping interval of powdery mildew resistance contained an allele of the $R w t 4$

89 NLR candidate identified in our study, in addition to the WTK. On functional testing by Lu et 90 al $(2020)^{16}$, the WTK, and not the NLR, was found to be necessary and sufficient to confer 91 resistance to powdery mildew and was designated as Pm24. Therefore, we tested both the 92 identified Rwt4 NLR candidate (Supplementary Fig. 11) and the linked Pm24 allele 93 (Supplementary Fig. 12) as candidates for Rwt4 using the Cadenza TILLING resource ${ }^{17}$. For 94 the NLR candidate, we identified four lines (two heterozygous and two homozygous) 95 carrying mutations predicted to cause premature stop codons and six additional lines (four 96 heterozygous and two homozygous) carrying mis-sense mutations predicted to have a 97 significant impact on tertiary structure (Table S6). Neither the homozygous nor any progeny 
of the heterozygous mutants for this candidate showed an increase in susceptibility relative to the wildtype Cadenza in either leaf or head assays with Br48+PWT4 (Supplementary Fig. 13). For the linked Pm24 allele, we tested three lines (one homozygous and two heterozygous) carrying mutations that result in premature stop codons (Table S6). In both the leaf and head assays of the homozygous line M0159 using Br48+PWT4, a clear increase in susceptibility compared to the wildtype was observed (Fig. 1g-h). In the leaf and head assays of the segregating progeny of heterozygous mutants (M0971 and M1103) using Br48+PWT4, those homozygous for the mutation were found to be susceptible while all others were resistant (Supplementary Fig. 14). These results show that as in the case of Pm24, the linked WTK, and not the identified NLR candidate, is required for resistance to $P$. oryzae expressing the PWT4 effector. The finding that WTK alleles, Pm24 and Rwt4, are involved in resistance to two unrelated fungal pathogens suggests that it may be a broad-spectrum component of disease resistance.

We developed KASP markers for Rwt3 and Rwt4 (Table S7) and validated them on the core 300 Watkins accessions (Table S8). Rwt3 is present only in 145 of the 193 Watkins accessions resistant to Br48+PWT3 (Fig. 2a, Table S8), while Rwt4 is present only in 136 of the 270 Watkins accessions resistant to Br48+PWT4 (Fig. 2b, Table S8). This suggests that there are other resistance genes in the Watkins panel recognising PWT3, PWT4 or additional effectors in Br48. We re-ran GWAS with the leaf assay disease phenotype of Br48+PWT4, restricted to the Watkins lines not containing Rwt4. Using Jagger as the reference genome, we obtained a clear peak on chromosome 1B in the region homeologous to that on $1 \mathrm{D}$ containing Rwt4 (Fig. 2e), indicating that Rwt4 has a homeologue on chromosome 1B that provides resistance to $P$. oryzae expressing the PWT4 effector. We followed the same protocol and reran the GWAS with the leaf assay disease phenotype of $\mathrm{Br} 48+P W T 3$, restricted to the Watkins lines not containing Rwt3. This identified a clear peak on chromosome 2A using Mattis as the reference genome (Fig. 2c) and another on chromosome 7A using Jagger as the reference genome (Fig. 2d). A resistance termed Rmg2 located on chromosome 7A has previously been identified in the cultivar Thatcher ${ }^{18}$ and a resistance termed $R m g 7$ has been reported on the distal region of the long arm of chromosome $2 \mathrm{~A}$ of tetraploid wheat ${ }^{19}$. In both instances the resistances were identified using the same isolate, $\mathrm{Br} 48$, as used in our work suggesting that the resistances identified on chromosomes $2 \mathrm{~A}$ and $7 \mathrm{~A}$ may correspond to $R m g 7$ and Rmg2 reported previously. Watkins lines carrying the 7A resistance showed similar levels of resistance to both $\mathrm{Br} 48$ and $\mathrm{Br} 48+P W T 3$ (Supplementary Fig. 15) indicating 
131 that this resistance is due to interaction with $\mathrm{Br} 48$ and supporting its characterisation as 132 Rmg2.

133 We designed a KASP-based marker for the Rwt4 1B homeologue (Tables S8, S9) which, 134 along with those for $R w t 3$ and $R w t 4-1 \mathrm{D}$, should enable wheat breeders to ensure that cultivars 135 contain resistance effective against PWT3 and PWT4 and therefore maintain host-specificity 136 barriers against Lolium and Avena pathotypes of $P$. oryzae. It was due to the lack of this 137 information that Rwt3 failed to make its way into elite cultivars such as Anahuac despite 138 being widely present in wheat landraces (Table S9), which was the probable cause of the 139 original wheat blast epidemic in Brazil (Fig. 3a). A future host jump of $P$. oryzae poses a high 140 risk of host range expansion of Triticum pathotypes of P. oryzae. This risk was illustrated in 141 the recent study of Inoue et al $(2021)^{20}$, which showed that the resistance conferred by $\operatorname{Rmg} 8$ 142 is suppressed by PWT4 and that the presence of Rwt4 in wheat prevents this suppression. $143 R m g 8$, along with $R m g 7$, recognises the effector $A V R-R m g 8$ and is one of the few reported 144 resistances that show effectiveness against Triticum pathotypes of $P$. oryzae at both the seedling and head stage ${ }^{21}$. If Triticum pathotypes acquire PWT4 from a future host jump, the resistance provided by $R m g 8$ would be lost (Fig. 3b). Therefore, it is important to ensure the presence of Rwt4 in wheat cultivars not only to prevent a future host jump but also to maintain the effectiveness of Rmg8 against wheat blast if such an event occurs. 


\section{Watkins panel configuration}

169 Using the SSR genotype data from Wingen et al $(2014)^{22}$, a core set of 300 genetically

170 diverse wheat landraces with spring growth habit were selected from the Watkins collection

171 (Supplementary Fig. 1, Table S2) along with 20 non-Watkins lines. The DNA was extracted

172 following a modified CTAB protocol ${ }^{23}$. The seeds of these lines are available from the

173 Germplasm Resources Unit (www.seedstor.ac.uk) under Wheat Resistance gene enrichment

174 (WREN) sequencing collection (WREN0001- WREN0320).

\section{Phenotyping of Ae. tauschii and Watkins panels with wheat blast isolates}

176 The M. oryzae pathotype Triticum (MoT) isolate $\mathrm{Br} 48$ and the transformed isolates

$177 \mathrm{Br} 48+P W T 3$ and $\mathrm{Br} 48+P W T 4^{7}$ were grown on complete medium agar (CMA). A conidial

178 suspension of $0.3-0.4 \times 10^{6}$ conidia per ml was used for all inoculations. Detached seedling

179 assays with the Ae. tauschii and Watkins panels were carried out as described by Goddard et

180 al $(2020)^{24}$ and scored for disease symptoms using a $0-6$ scale (Supplementary Fig. 2, 3 and

181 6; Table S3, S5). Resistance at the heading stage was assessed according to Goddard et al

$182(2020)^{24}$. Heads of Ae. tauschii and wheat were scored using a $0-6$ scale (Supplementary

183 Fig. 2e and 2f, respectively).

\section{Bait library design for the Watkins panel}

185 Two bait libraries were used for the capture of the immune receptors from the Watkins panel

186 (i) NLR Triticeae bait library V3 (https://github.com/steuernb/MutantHunter/), including 275

187 genes conserved in grasses ${ }^{25}$ and (ii) A new bait library which included NLRs extracted from

188 the genomes of T. turgidum cv. Svevo and cv. Kronos and T. dicoccoides cv. Zavitan and

189 only those genes that had $<50 \%$ coverage by previously designed baits were used. To remove

190 redundancies, NLR sequences were passed through CD-HIT (v4.6.8-2017-0621 -c 0.9 -G 0 -

191 aS 0.9 -p 1). This bait design also included wheat domestication genes VRN1A (AY747598),

192 Wxl (AY050174), Q (AY702956), Rht-bl (JX993615), Rht-dl (HE585643), NAM-B1

193 (MG587710) as well as wheat orthologs of known immune signalling components ICS1,

194 NPR1, NDR1, EDS1, PAD4, SRFR1, SAG101, RAR1, SGT1, HSP90.2, HSP90.4, RIN4,

195 ADR1 and PBS1 extracted through BioMart (Table S1, Additional File 1). The bait probes

196 were designed by Arbor Bioscience and filtered with their Repeat Mask pipeline which 
removed the baits that were $>50 \%$ Repeat Masked and any non-NLR baits with $>3$ hits in the

198 wheat genome. To balance for the low copy number genes, baits derived from domestication

199 genes were multiplied 10x and those derived from immune signalling genes were $3 \mathrm{x}$

200 compared to the baits derived from NLRs.

201 Library construction and sequencing of the Watkins panel

202 Illumina libraries with an average insert size of $700 \square$ bp were enriched by Arbor Biosciences,

203 Michigan, USA, as previously described ${ }^{26}$, and sequenced on an Illumina HiSeq with either

204150 or $250 \mathrm{PE}$ reads at Novogene, China to generate an average of $3.82 \mathrm{~Gb}$ per accession

205 (Table S2). The raw reads were trimmed using Trimmomatic v0.2 $2^{27}$ and de novo assembled

206 with the CLC Assembly Cell (http://www.clcbio.com/products/clc-assembly-cell/) using

207 word size (-w $\square=\square 64$ ) with standard parameters.

208 Generating Watkins $\boldsymbol{k}$-mer presence/absence matrix and its phylogeny

209 A presence/absence matrix of $k$-mers $(\mathrm{k} \square=\square 51)$ was constructed from trimmed raw data 210 using Jellyfish ${ }^{28}$ as described in Arora et al (2019) ${ }^{13} . k$-mers occurring in less than four 211 accessions or in all but three or fewer accessions were removed during the construction of the 212 matrix. From the $k$-mer matrix generated with Watkins RenSeq data, 5310 randomly 213 extracted $k$-mers were used to build a UPGMA (unweighted pair group method with 214 arithmetic mean) tree with 100 bootstraps.

\section{$215 \boldsymbol{k}$-mer based association mapping}

216 For the reference genomes of T. aestivum - Chinese Spring ${ }^{29}$, Jagger and Mattis ${ }^{30}$ - and of Ae.

217 tauschii AY61 ${ }^{31}$, NLRs were predicted using NLR-Annotator ${ }^{32}$ and their sequences along

218 with $3 \mathrm{~kb}$ sequence from both upstream and downstream region (if available) were extracted

219 using samtools (version 1.9) to create the corresponding reference NLR assemblies. The

220 disease phenotypes were averaged across the replicates after removing the non-numerical

221 values and the mean phenotype scores multiplied by -1 so that a higher value represents a

222 higher resistance. For those $k$-mers of a reference NLR assembly whose presence/absence in 223 the panel correlates with the phenotype, that is, the absolute value of Pearson's correlation 224 obtained was higher than 0.1 , a p-value was assigned using linear regression while taking the 225 three most significant PCA dimensions as covariates to control for the population structure. A 226 stringent cut-off of 8 , based on Bonferroni-adjustment ${ }^{14}$ to a $p$-value of 0.05 , was chosen for 227 Watkins RenSeq association mapping, while a cut-off of 7 was chosen for Ae. tauschii L2 228 RenSeq association mapping (Supplementary Fig. 7).

\section{In silico gene structure prediction}


230 The Rwt3 NLR candidate gene transcript is 5,937 bp. Only one of the 15 annotated exons

231 (grey colored exon in Fig. 1c) appears to be translated into protein. This exon encodes a

232 protein of 1069 amino acids with a coiled-coil domain, an NB-ARC domain and several

233 leucine rich repeats (LRRs) motifs at the C-terminus (Supplementary Fig. 4). The Rwt4 NLR

234 candidate gene is 3,117 bp with three exons. The predicted protein of 1038 amino acids

235 contain domains with homology to a coiled-coil (CC) domain, two NB-ARC domains and

236 two LRR at the C-terminus (Supplementary Fig. 11). The Rwt4 WTK candidate has an open

237 reading frame of $2,751 \mathrm{bp}$ which has eleven predicted exons that encode a protein of 916

238 amino acids with putative tandem protein kinase domains (Fig. 1f; Supplementary Fig. 12).

239 Domains were predicted by NCBI and Pfam databases. The gene structure of both Rwt3 and

240 Rwt4 NLR candidate genes was consistent with that predicted using cDNA RenSeq data of

241 Watkins lines.

242 Identification and phenotyping of Cadenza TILLING mutants to test the function of

$243 \quad$ Rwt4

244 Cadenza TILLING lines ${ }^{17}$ for the NLR candidate for Rwt4 were identified within the Plant

245 Ensembl database for the gene TraesCS1D02G059000

246 (http://plants.ensembl.org/Triticum_aestivum/Gene/). Lines containing mutations leading to

247 premature stop codons and those for which the 'sorting intolerant from tolerant' (SIFT) score

248 was 0.0 or 0.01 were selected for phenotyping. For the Rwt4 kinase candidate gene, Cadenza

249 TILLING lines were identified for the gene TraesCS1D02G058900. Details of the mutations

250 present in the Cadenza TILLING lines is provided in the Table S6.

251 Identification and phenotyping of Jagger TILLING mutants to test the function of $R w t 3$

252 For selecting mutations in the Rwt3 candidate gene (TraesCS1D02G029900), TILLING was

253 performed in wheat cultivar Jagger ${ }^{15}$ using genome specific primer pairs (Supplementary Fig.

$2549 \mathrm{a}-\mathrm{d})$. The effects of the mutations on the predicted protein were analysed using SnapGene $\mathbb{}$

255 software (version 5.0.7 from GSL Biotech). The effects of missense mutations were

256 determined using PROVEAN (Protein Variation Effect Analyzer) v1.1 software ${ }^{33}$. Selected

257 lines were phenotyped as described above. Details of the mutations is provided in Table S6.

258 KASP analysis and sequencing of TILLING lines to confirm mutations

259 Kompetitive Allele-Specific PCR (KASP) (LGC Genomics) was performed to confirm

260 mutations where suitable PCR primers could be designed. Alternatively, the region

261 containing the mutation was amplified and purified products were sequenced by Eurofins

262 Genomics. Sequence analysis was performed with Geneious Prime software.

263 Anahuac DNA preparation, sequencing, and assembly to check presence of $R$ wt3 gene 
264 To confirm that Anahuac is a non-carrier of $R w t 3$, we captured its NLR complement using

265 the bait libraries described above. The Rwt3 NLR candidate was absent in the CLC assembly

266 generated as described above.

267 KASP marker design to detect $\boldsymbol{R} w t 3$ and $\boldsymbol{R} w \boldsymbol{t} 4$ in wheat cultivars and Watkins collection

268 The regions differentiating resistant and susceptible alleles of Rwt4 from the Ae. tauschii L2

269 panel were used to design KASP markers. The KASP marker discriminated between resistant

270 and susceptible accessions in Ae. tauschii L2 panel but did not distinguish reliably between

271 resistant and susceptible lines in the Watkins panel. The resistant allele of Rwt4 was the same

272 in both the Ae. tauschii L2 and the Watkins panels but the susceptible allele of Rwt4 in the

273 Watkins panel originated from Ae. tauschii L3 and not Ae. tauschii L2. This is consistent with

274 the multi-lineage hybridisation hypothesis proposed in Gaurav et al 2021 ${ }^{14}$. A new marker

275 was designed by comparing the common resistant allele with susceptible alleles from both the

276 Ae. tauschii L2 and Watkins panels (Table S7) that successfully distinguished between the

277 resistant and susceptible alleles in the wheat lines (Table S8).

278 We used the D-genomes of 11 chromosome-scale wheat assemblies ${ }^{30}$ to fetch the D-genome 279 susceptible allele of $R w t 3$ and designed KASP markers (Table S7). The marker distinguished 280 resistant from susceptible lines and had a high correlation with presence-absence scored with 281 in silico markers (Table S8). KASP markers were tested on the entire Watkins panel ( 900)

282 to understand the distribution of these genes in the landrace collection (Table S9).

\section{Characterisation of the resistance identified on chromosome 7A}

284 A set of Watkins lines were genotyped as carrying either Rwt3 or the 7A resistance or having 285 neither or both resistances. All accessions were phenotyped in leaf assays using isolates $\mathrm{Br} 48$ 286 and $\mathrm{Br} 48+P W T 3$. Accessions lacking either resistance were susceptible to both isolates 287 (Supplementary Fig. 15). Accessions carrying either the 7A resistance alone or both the 7A 288 resistance and $R w t 3$ showed similar level of resistance to both $\mathrm{Br} 48$ and $\mathrm{Br} 48+P W T 3$.

\section{Acknowledgements}

291 The high-performance computing resources and services used in this work were supported by 292 the Norwich Bioscience Institutes Partnership (NBIP) Computing infrastructure for Science 293 (CiS) group alongside the Earlham Institute (EI) scientific computing group. We are grateful 294 to the John Innes Centre (JIC) Horticultural Services for plant husbandry; EI for providing 295 open access to the Kronos genome. This research was financed by the Biotechnology and 296 Biological Sciences Research Council (BBSRC) Designing Future Wheat Cross-Institute 
297 Strategic Programme to BBHW and PN (BBS/E/J/000PR9780); a John Innes Centre Institute

298 Strategic Grant to BW; Science, Technology \& Innovation Funding Authority (STDF),

299 Egypt-UK Newton-Mosharafa Institutional Links award, Project ID (30718) to AFE and

300 BBHW; the Gordon and Betty Moore Foundation through grant GBMF4725 to the Two

301 Blades Foundation; and the Gatsby Charitable Foundation to JDGJ; National Science

302 Foundation (Award\#1943155) and USDA NIFA (Award\#2020-67013-32558 and 2020-

303 67013-31460) to NR and VT; European Research Commission grant (ERC-2016-STG-

304 716233-MIREDI) to KVK and BBSRC Norwich Research Park Doctoral Training Grant

305 (BB/M011216/1) for supporting EB.

306 Author contributions

307 This work was conceived by PN, JC and BBHW. Watkins panel configuration, DNA

308 extraction and sequence acquisition (BBHW, LW, MFM, RA, SA, GY, AFE, JDGJ). Bait

309 library design (KVK, EB, BS), $k$-mer matrix construction and association mapping (SA, KG),

310 candidate genes discovery and analysis (SA, KG), Phylogenetic analysis (SA, KG), Blast

311 isolates (YT, SAs), Phenotyping of diversity panels and TILLING mutants (AS, RG, TH, PN,

$312 \mathrm{CC}, \mathrm{MHN}$ ), KASP marker design and analysis (SA, AS, KG, PN), Jagger mutants

313 identification (VT, ASc, NR), Mutant confirmation and segregation (AS, RG, PN), cDNA

314 RenSeq data (SA, AFE), Drafted manuscript (SA, PN, KG, RG, AS, VT, YT, ASc, NR, KK)

315 and designed figures (SA, PN, KG, RG, AS, ASc, LW).

\section{Competing interests}

317 The authors declare no competing interests. 

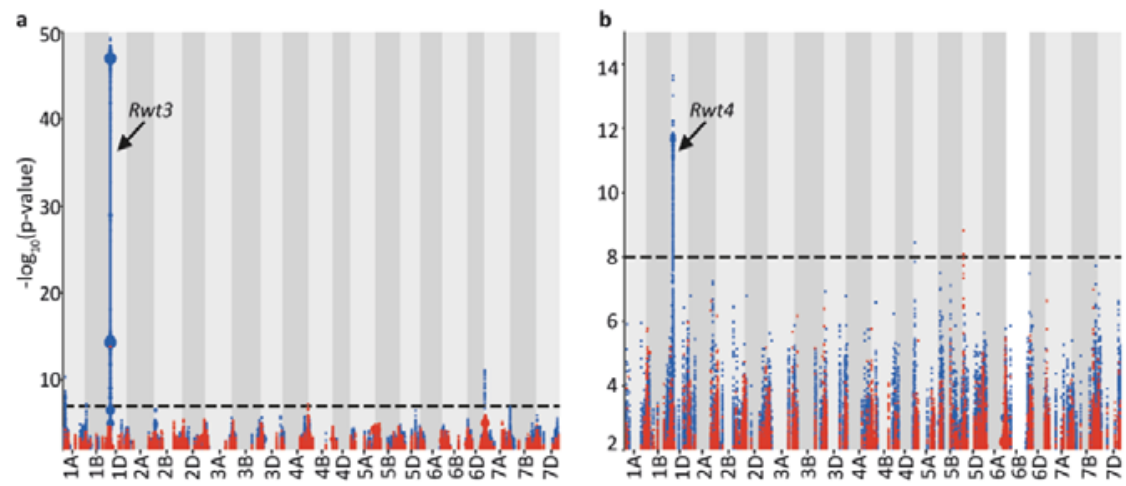

c ATG Exon1 Exon15

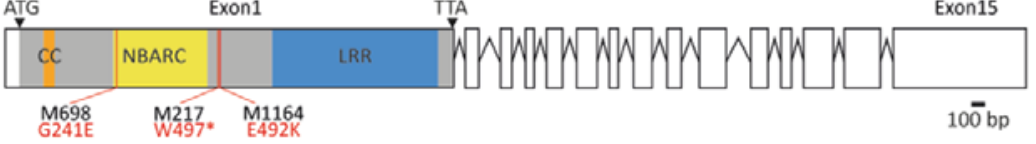

d
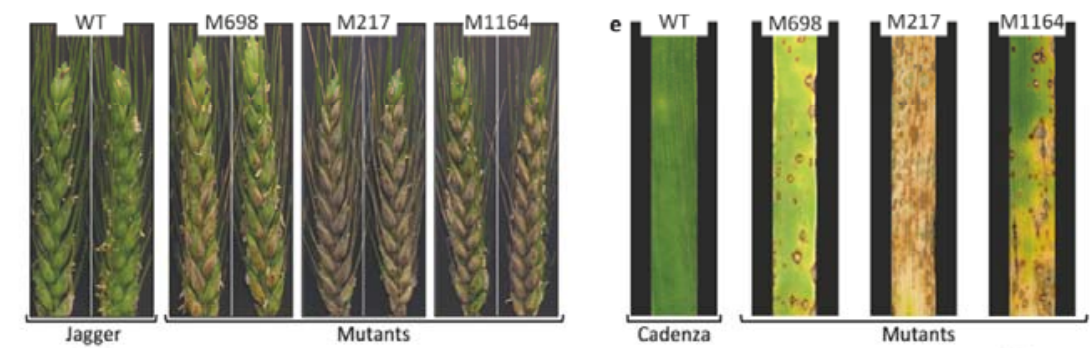

$\mathbf{f}$

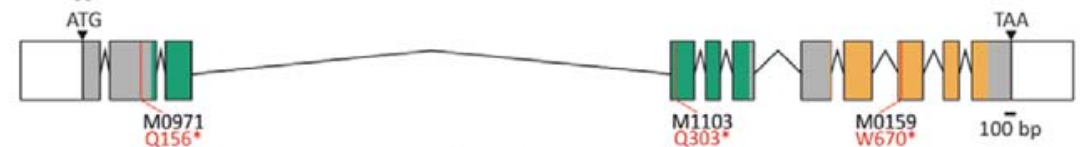

g
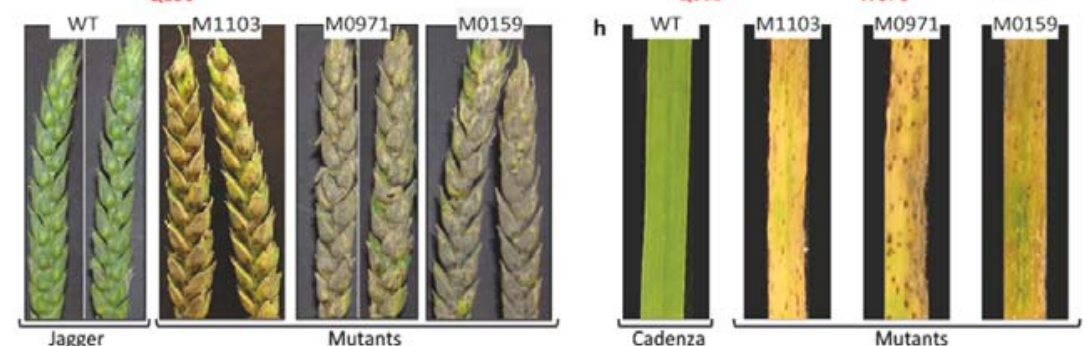

323 Figure 1| Genetic identification of the candidate genes recognising host-specific 324 avirulence effectors of the blast fungus by $\boldsymbol{k}$-mer-based association mapping on an $\boldsymbol{R}$ gene enriched sequencing panel of wheat landraces. $k$-mers associated with resistance to (a) Br48+Pwt3 mapped to Chinese Spring, and (b) Br48+Pwt4 mapped to Jagger. Points on the $\mathrm{y}$-axis depict $k$-mers positively associated with resistance in blue and negatively associated with resistance in red. Point size is proportional to the number of $k$-mers. (c) Structure of the NLR candidate gene for Rwt3. The predicted 1069 amino acids protein has domains with homology to a coiled-coil (CC), nucleotide-binding (NBARC) and leucine-rich repeats (LRR). Wheat blast (d) head and (e) detached leaf assays for the Rwt3 Jagger mutants and wild type with $\mathrm{Br} 48+P W T 3$. (f) Structure of the candidate gene for Rwt4. The predicted protein of 916 amino acids has domains with homology to a wheat tandem kinase (shown with green and orange colors). Wheat blast (g) head and (h) detached leaf assays for the Rwt4 Cadenza mutants and wild type with Br48+PWT4. 


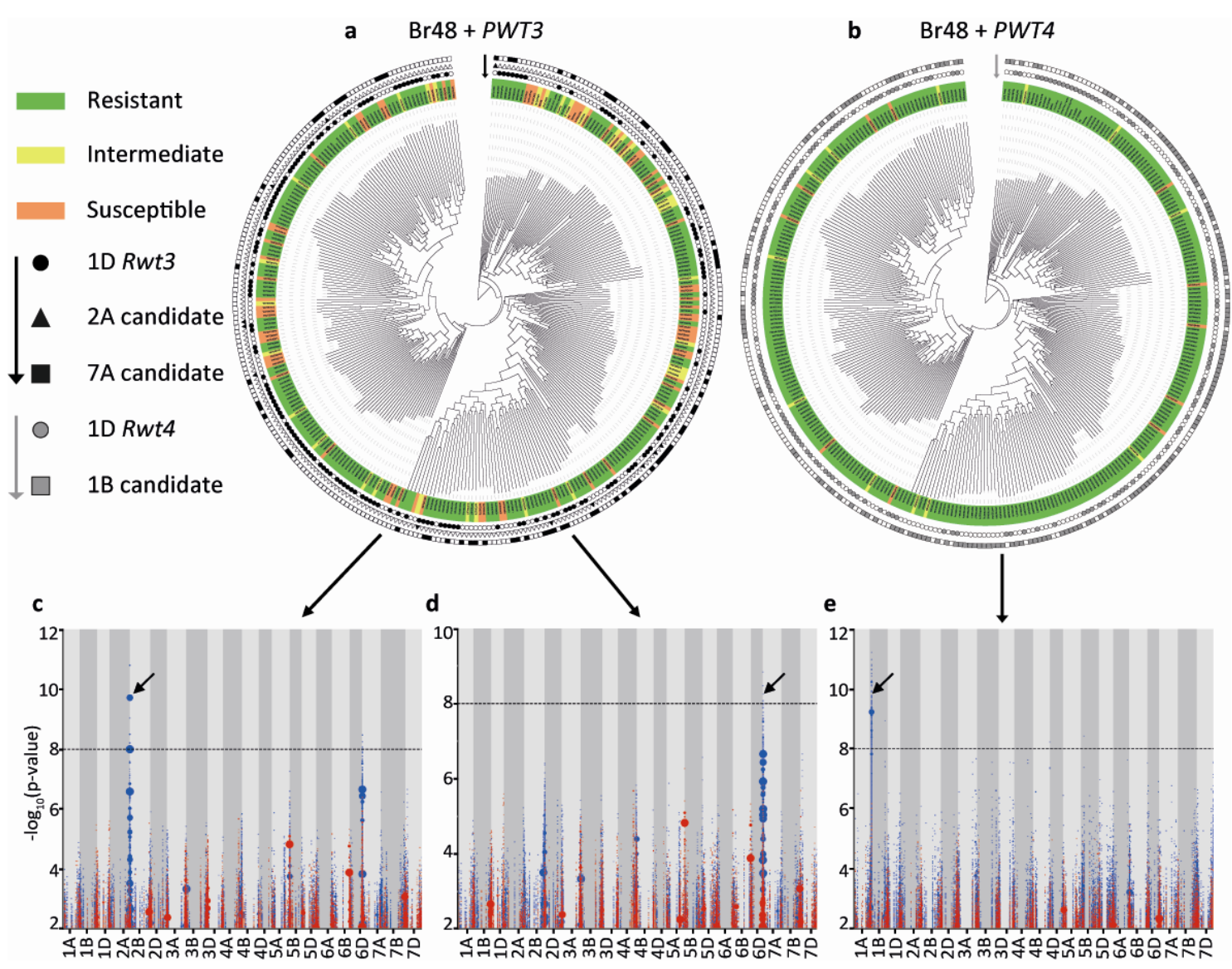

337 Figure 2 | Additional resistances to blast fungus and their distribution in the diversity

338 panel of wheat landraces. (a)-(b) $k$-mer-based phylogeny of wheat landraces showing the 339 phenotype of an accession after inoculation with: (a) $\mathrm{Br} 48+P w t 3$ and (b) $\mathrm{Br} 48+P w t 4$, and the 340 presence of the respective candidate resistance genes. Phenotype of an accession after 341 inoculation with a blast isolate is indicated by the color used to highlight the label of that 342 accession, while the presence and absence of allele-specific polymorphisms is indicated by 343 filled symbols with black/grey or white, respectively. $k$-mers significantly associated with 344 resistance to $\mathrm{Br} 48+P w t 3$ in the absence of the $R w t 3$ candidate gene on chromosome 1D leads 345 to the identification of a resistance on (c) chromosome 2A when mapped to the assembly of 346 wheat cultivar SY Mattis, and (d) chromosome 7A when mapped to wheat cultivar Jagger. (e) $347 k$-mers significantly associated with resistance to $\mathrm{Br} 48+P w t 4$ in the absence of $R w t 4$ candidate 348 gene on chromosome 1D leads to the identification of a resistance on a region of chromosome 349 1B containing the homeologue of $R w t 4$. 


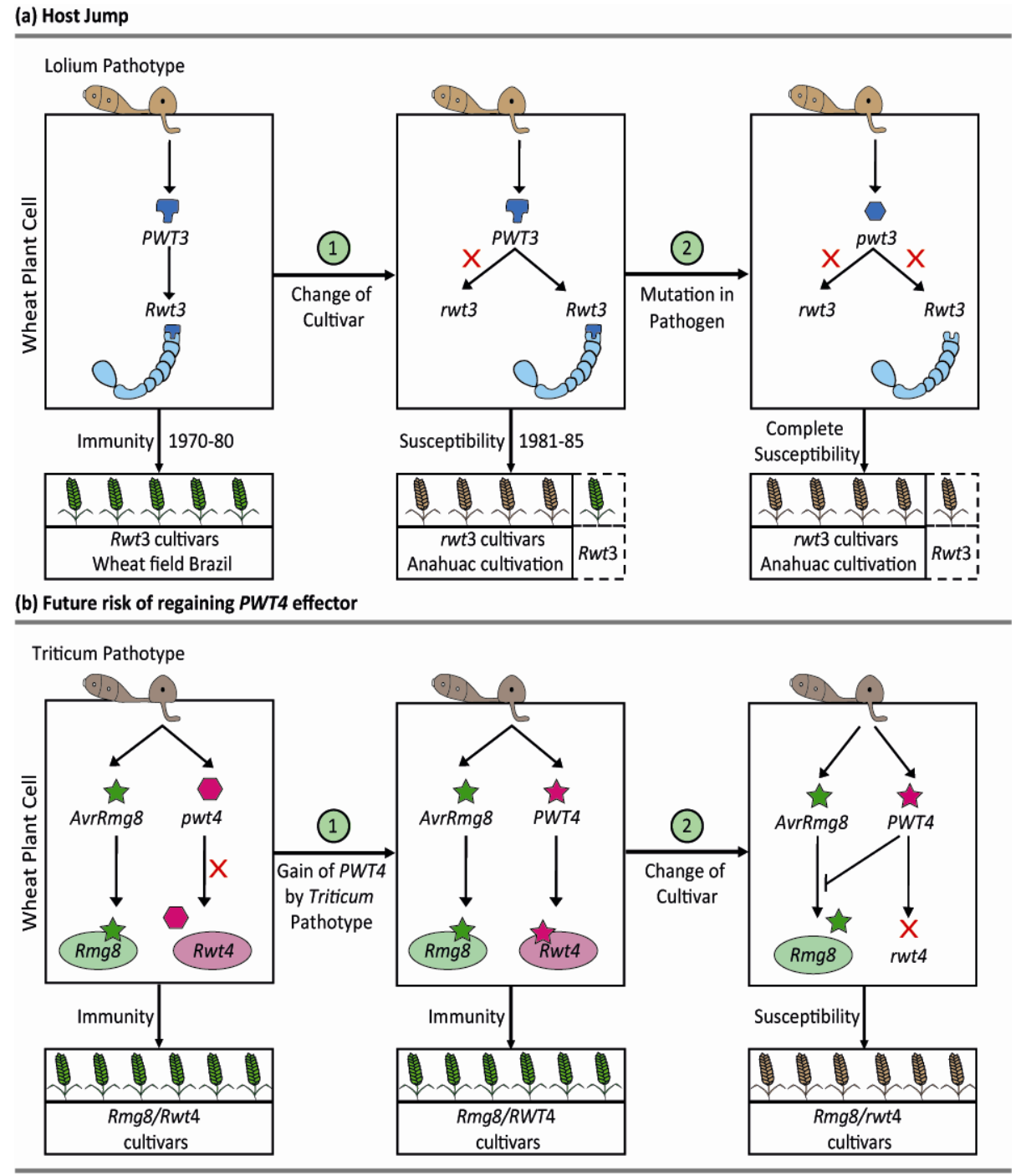

351 Figure 3 | A possible working model of host jump of blast fungus from Lolium to wheat
352 and a future risk associated with the reacquisition of PWT4. (a) (i) A schematically drawn wheat cell of a cultivar carrying Rwt3 attacked by a Lolium isolate of the blast fungus. The PWT3 effector is recognized by Rwt3 thus preventing the Lolium isolate from infecting wheat. (ii) Widespread cultivation of cultivars lacking $R w t 3$ (or having the susceptible allele, rwt3) allowed the Lolium isolate to colonize wheat. (iii) The colonizing blast population further expanded the host range by losing PWT3 (or gaining the non-interacting effector, pwt3) through mutation or recombination. (b) (i) A schematically drawn wheat cell of a cultivar carrying Rmg8 and Rwt4 attacked by a Triticum isolate carrying AvrRmg8. The AvrRmg 8 effector is recognized by Rmg8, thus preventing Triticum isolate from infecting the cultivar. (ii) Gain of Triticum isolates gain the PWT4 effector due to a future host jump but are still not able to infect the cultivars carrying Rwt4 (iii) However, cultivars lacking Rwt4 (or having the susceptible allele, rwt4) will be susceptible to the Triticum isolate carrying both AvrRmg 8 and PWT4 even if the cultivar carries Rmg 8 because in the absence of Rwt4, PWT4 suppresses the recognition of AvrRmg 8 by Rmg8. Therefore, the Triticum pathotype will be able to further expand its host range. 


\section{References}

368 1. Panstruga, R. \& Moscou, M. J. What is the molecular basis of nonhost resistance? Mol.

369 Plant-Microbe Interact. 33, 1253-1264 (2020).

370 2. Igarashi S, Utiamada C M, Igarashi L C, Kazuma A H, L. R. S. Pyricularia in wheat: 1. 371 Occurrence of Pyricularia sp. in Parana State. Fitopatol Bras 11, 351-352 (1986).

372 3. Cruz, C. D. \& Valent, B. Wheat blast disease: danger on the move. Trop. Plant Pathol. $373 \quad$ 42, 210-222 (2017).

374 4. Malaker, P. K. et al. First Report of Wheat Blast Caused by Magnaporthe oryzae 375 Pathotype triticum in Bangladesh. Plant Dis. 100, 2330 (2016).

376 5. Tembo, B. et al. Detection and characterization of fungus (Magnaporthe oryzae

6. Singh, P. K. et al. Wheat Blast: A Disease Spreading by Intercontinental Jumps and Its pathotype Triticum) causing wheat blast disease on rain-fed grown wheat (Triticum aestivum L.) in Zambia. PLoS One 15, e0238724 (2020).

7. Inoue, Y. et al. Evolution of the wheat blast fungus through functional losses in a host specificity determinant. Science (80-. ). 357, 80-83 (2017).

8. Takabayashi, N., Tosa, Y., Oh, H. S. \& Mayama, S. A Gene-for-Gene Relationship Underlying the Species-Specific Parasitism of Avena/Triticum Isolates of Magnaporthe grisea on Wheat Cultivars. http://dx.doi.org/10.1094/PHYTO.2002.92.11.1182 92, 1182-1188 (2007).

9. Vy, T. T. P. et al. Genetic analysis of host-pathogen incompatibility between Lolium isolates of Pyricularia oryzae and wheat. J. Gen. Plant Pathol. 80, 59-65 (2014). single blasted wheat plant in the United States. Plant Dis. 101, 684-692 (2017). 
393

394

395

396

12. Hirata, K., Tosa, Y., Nakayashiki, H. \& Mayama, S. Significance of PWT4-Rwt4 interaction in the species specificity of Avena isolates of Magnaporthe oryzae on wheat. J. Gen. Plant Pathol. 71, 340-344 (2005).

13. Arora, S. et al. Resistance gene cloning from a wild crop relative by sequence capture and association genetics. Nat. Biotechnol. 2019372 37, 139-143 (2019).

14. Gaurav, K. et al. Population genomic analysis of Aegilops tauschii identifies targets for bread wheat improvement. Nat. Biotechnol. 2021 1-10 (2021). doi:10.1038/s41587-021-01058-4

15. Rawat, N. et al. A TILLING Resource for Hard Red Winter Wheat Variety Jagger. Crop Sci. 59, 1666-1671 (2019).

16. Lu, P. et al. A rare gain of function mutation in a wheat tandem kinase confers resistance to powdery mildew. Nat. Commun. 2020111 11, 1-11 (2020).

17. Krasileva, K. V. et al. Uncovering hidden variation in polyploid wheat. Proc. Natl. Acad. Sci. U. S. A. 114, E913-E921 (2017).

18. Zhan, S. W., Mayama, S. \& Tosa, Y. Identification of two genes for resistance to Triticum isolates of Magnaporthe oryzae in wheat. Genome 51, 216-221 (2008).

19. Tagle, A. G., Chuma, I. \& Tosa, Y. Rmg7, a New Gene for Resistance to Triticum Isolates of Pyricularia oryzae Identified in Tetraploid Wheat. Phytopathology 105, 495-499 (2015).

20. Inoue, Y., Vy, T. T. P., Tani, D. \& Tosa, Y. Suppression of wheat blast resistance by an effector of Pyricularia oryzae is counteracted by a host specificity resistance gene in wheat. New Phytol. 229, 488-500 (2021).

21. Anh, V. L. et al. Rmg8, a new gene for resistance to Triticum isolates of Pyricularia oryzae in hexaploid wheat. Phytopathology 105, 1568-1572 (2015).

22. Wingen, L. U. et al. Establishing the A. E. Watkins landrace cultivar collection as a resource for systematic gene discovery in bread wheat. Theor. Appl. Genet. 127, 18311842 (2014). 
23. Yu, G., Hatta, A., Periyannan, S., Lagudah, E. \& Wulff, B. B. H. Isolation of Wheat Genomic DNA for Gene Mapping and Cloning. Methods Mol. Biol. 1659, 207-213 (2017).

24. Goddard, R. et al. Dissecting the genetic basis of wheat blast resistance in the Brazilian wheat cultivar BR 18-Terena. BMC Plant Biol. 20, 1-15 (2020).

25. Marcussen, T. et al. Ancient hybridizations among the ancestral genomes of bread wheat. Science (80-. ). 345, (2014).

26. Steuernagel, B., Witek, K., Jones, J. D. G. \& Wulff, B. B. H. MutRenSeq: A Method for Rapid Cloning of Plant Disease Resistance Genes. Methods Mol. Biol. 1659, 215229 (2017).

27. Bolger, A. M., Lohse, M. \& Usadel, B. Trimmomatic: a flexible trimmer for Illumina sequence data. Bioinformatics 30, 2114-2120 (2014).

28. Marçais, G. \& Kingsford, C. A fast, lock-free approach for efficient parallel counting of occurrences of k-mers. Bioinformatics 27, 764-770 (2011).

29. International Wheat Genome Sequencing Consortium (IWGSC), T. I. W. G. S. C. et $a l$. Shifting the limits in wheat research and breeding using a fully annotated reference genome. Science 361, eaar7191 (2018).

30. Walkowiak, S. et al. Multiple wheat genomes reveal global variation in modern breeding. Nat. 20205887837 588, 277-283 (2020).

31. Zhou, Y. et al. Introgressing the Aegilops tauschii genome into wheat as a basis for cereal improvement. Nat. Plants 2021 76 7, 774-786 (2021).

32. Steuernagel, B. et al. The NLR-Annotator Tool Enables Annotation of the Intracellular Immune Receptor Repertoire. Plant Physiol. 183, 468-482 (2020).

33. Choi, Y., Sims, G. E., Murphy, S., Miller, J. R. \& Chan, A. P. Predicting the functional effect of amino acid substitutions and indels. PLoS One 7, (2012). 